\title{
Patients with immune-mediated inflammatory diseases receiving cytokine inhibitors have low prevalence of SARS-CoV-2 infection
}

David Simon

Department of Internal Medicine 3, Friedrich-Alexander University (FAU) Erlangen-Nuremberg and Universitätsklinikum Erlangen, Erlangen, Germany

Koray Tascilar

Department of Internal Medicine 3, Friedrich-Alexander University (FAU) Erlangen-Nuremberg and Universitätsklinikum Erlangen, Erlangen, Germany

Gerhard Krönk

Department of Internal Medicine 3, Friedrich-Alexander University (FAU) Erlangen-Nuremberg and Universitätsklinikum Erlangen, Erlangen, Germany

Arnd Kleyer

Department of Internal Medicine 3, Friedrich-Alexander University (FAU) Erlangen-Nuremberg and Universitätsklinikum Erlangen, Erlangen, Germany

Mario Zaiss

Department of Internal Medicine 3, Friedrich-Alexander University (FAU) Erlangen-Nuremberg and Universitätsklinikum Erlangen, Erlangen, Germany

\section{Franz Heppt}

Deutsches Zentrum fuer Immuntherapie (DZI), FAU Erlangen-Nuremberg and Universitätsklinikum Erlangen, Erlangen, Germany

\section{Christine Meder}

Deutsches Zentrum fuer Immuntherapie (DZI), FAU Erlangen-Nuremberg and Universitätsklinikum Erlangen, Erlangen, Germany

Raja Atreya

Deutsches Zentrum fuer Immuntherapie (DZI), FAU Erlangen-Nuremberg and Universitätsklinikum Erlangen, Erlangen, Germany

\section{Entcho Klenske}

Deutsches Zentrum fuer Immuntherapie (DZI), FAU Erlangen-Nuremberg and Universitätsklinikum Erlangen, Erlangen, Germany

Peter Dietrich

Deutsches Zentrum fuer Immuntherapie (DZI), FAU Erlangen-Nuremberg and Universitätsklinikum Erlangen, Erlangen, Germany

Abdullah Abdullah

Deutsches Zentrum fuer Immuntherapie (DZI), FAU Erlangen-Nuremberg and Universitätsklinikum Erlangen, Erlangen, Germany

Thorsten Kliem

Deutsches Zentrum fuer Immuntherapie (DZI), FAU Erlangen-Nuremberg and Universitätsklinikum Erlangen, Erlangen, Germany

Giulia Corte

Department of Internal Medicine 3, Friedrich-Alexander University (FAU) Erlangen-Nuremberg and Universitätsklinikum Erlangen, Erlangen, Germany

Harriet Morf

Department of Internal Medicine 3, Friedrich-Alexander University (FAU) Erlangen-Nuremberg and Universitätsklinikum Erlangen, Erlangen, Germany

Moritz Leppkes

Deutsches Zentrum fuer Immuntherapie (DZI), FAU Erlangen-Nuremberg and Universitätsklinikum Erlangen, Erlangen, Germany

Andreas Kremer

Deutsches Zentrum fuer Immuntherapie (DZI), FAU Erlangen-Nuremberg and Universitätsklinikum Erlangen, Erlangen, Germany

Andreas Ramming

Department of Internal Medicine 3, Friedrich-Alexander University (FAU) Erlangen-Nuremberg and Universitätsklinikum Erlangen, Erlangen, Germany

Milena Pachowsky

Department of Internal Medicine 3, Friedrich-Alexander University (FAU) Erlangen-Nuremberg and Universitätsklinikum Erlangen, Erlangen, Germany

Florian Schuch

Rheumatology Clinical Practice Erlangen, Erlangen, Germany

\section{Monika Ronneberger}

Rheumatology Clinical Practice Erlangen, Erlangen, Germany

Stefan Kleinert

Rheumatology Clinical Practice Erlangen, Erlangen, Germany

Clara Maier

Institute of Clinical and Molecular Virology, FAU Erlangen-Nuremberg and Universitätsklinikum Erlangen, Erlangen, Germany

\section{Axel Hueber}

Department of Internal Medicine 3, Friedrich-Alexander University (FAU) Erlangen-Nuremberg and Universitätsklinikum Erlangen, Erlangen, Germany

\section{Karin Manger}

Rheumatology Practice Bamberg, Bamberg, Germany

Bernhard Manger

Department of Internal Medicine 3, Friedrich-Alexander University (FAU) Erlangen-Nuremberg and Universitätsklinikum Erlangen, Erlangen, Germany Carola Berking 
Deutsches Zentrum fuer Immuntherapie (DZI), FAU Erlangen-Nuremberg and Universitätsklinikum Erlangen, Erlangen, Germany

Matthias Tenbusch

Institute of Clinical and Molecular Virology, FAU Erlangen-Nuremberg and Universitätsklinikum Erlangen, Erlangen, Germany

Klaus Überla

Institute of Clinical and Molecular Virology, FAU Erlangen-Nuremberg and Universitätsklinikum Erlangen, Erlangen, Germany

Michael Sticherling

Deutsches Zentrum fuer Immuntherapie (DZI), FAU Erlangen-Nuremberg and Universitätsklinikum Erlangen, Erlangen, Germany

Markus F. Neurath

Deutsches Zentrum fuer Immuntherapie (DZI), FAU Erlangen-Nuremberg and Universitätsklinikum Erlangen, Erlangen, Germany

Georg Schett ( $\nabla$ georg.schett@uk-erlangen.de)

Department of Internal Medicine 3, Friedrich-Alexander University (FAU) Erlangen-Nuremberg and Universitätsklinikum Erlangen, Erlangen, Germany

\section{Research Article}

Keywords: Immune-mediated inflammatory diseases, inflammatory cytokine inhibitors, COVID-19

Posted Date: June 10th, 2020

DOI: https://doi.org/10.21203/rs.3.rs-34550/v1

License: @ (i) This work is licensed under a Creative Commons Attribution 4.0 International License. Read Full License

Version of Record: A version of this preprint was published at Nature Communications on July 24th, 2020. See the published version at https://doi.org/10.1038/s41467-020-17703-6. 


\section{Abstract}

Immune-mediated inflammatory diseases (IMIDs) of the joints, gut and skin are treated with inhibitors of inflammatory cytokines. These cytokines are involved in the pathogenesis of coronavirus disease 2019 (COVID-19). Investigating anti-SARS-CoV-2 antibody responses in IMIDs we observed a significantly reduced incidence of SARS-CoV-2 infection in IMID patients treated with cytokine inhibitors compared to patients receiving no such inhibitors and two healthy control populations, despite similar social exposure. Hence, cytokine inhibitors seem to at least partially protect from SARS-CoV-2 infection.

Authors David Simon and Koray Tascilar contributed equally to this work.

Authors Markus F. Neurath and Georg Schett share senior authorship.

\section{Main Text}

In December 2019, a new coronavirus, named Severe Acute Respiratory Syndrome Corona Virus 2 (SARS-CoV-2) emerged in Wuhan leading to a cluster of respiratory infections [1]. This coronavirus disease 2019 (COVID-19) rapidly spreads worldwide and the virus increasingly challenges patient groups with enhanced infection risk. The severity of COVID-19 is highly heterogeneous and is modulated by yet to be identified host factors. While most individuals experience no or rather mild respiratory symptoms, COVID-19 can also lead to severe pneumonia with acute respiratory distress syndrome (ARDS) and death [2]. Age, smoking (which increases expression of ACE2, the epithelial receptor for SARS-CoV-2) and certain comorbidities, such as diabetes mellitus, arterial hypertension and obstructive lung diseases, are associated with severe courses of COVID-19 [2].

In the context of COVID-19, patients with IMIDs are of particular interest, as these patients are characterized by intrinsic immune dysfunction as well as immune modulatory treatments that may enhance infection risk. IMIDs often affect the inner and outer barriers of the body such as the joints (rheumatoid arthritis, spondyloarthritis), gut (inflammatory bowel disease) and skin (psoriasis) [3]. Therapeutic interventions for IMIDs target inflammatory cytokines, such as TNF-a, IL -6 and IL-17 that are both involved in the physiological and pathological host response elicited by SARS-CoV-2 [4]. We therefore questioned whether IMID patients receiving cytokine inhibitors might be at higher risk for SARS-CoV-2 infection.

We documented respiratory and other infectious symptoms as well as social behavior during the outbreak of COVID-19 in Europe in February up to April 2020 for a period of 12 weeks. This analysis was performed in patients with IMIDs receiving continuous cytokine blockade for their underlying disease $(N=534)$, in patients with IMIDs receiving no cytokine inhibition $(N=259)$, in health care professionals involved in the treatment of these patients (health-care [HC] control, $\mathrm{N}=285$ ) and in a cohort of healthy control subjects unrelated to health care ([5]; non-health care [NHC] control, $N=971)$ from the same region. Details of IMID patients and the controls are depicted in Figure 1A. To test for exposure to SARS-CoV-2 we made use of recent insights into the anti- SARS-CoV-2 immune response: Spike proteins and the nucleocapsid are known as the main antigens of Corona viruses [6,7]. The S1 domain of the SARS-CoV-2 spike protein has been shown to provide good specificity with limited cross-reactivity to antibodies raised during infections with the four endemic coronaviruses [8]. We therefore used an S1 domain-based antibody ELISA for the initial testing and confirmed all positive samples by determining the antibody response to the SARS-CoV-2 nucleocapsid (N) protein.

Anti-SARS-CoV-2 IgG defined as an OD450nm of $\geq 0.8$ in the lgG antibody test against the spike protein domain $\mathrm{S} 1$ was found in $2.26 \%$ (prevalence [95\% $\mathrm{Cl}$ ] 0.023 [0.014 to 0.034]) of the subjects of the NHC control cohort (Figure 1B). Age-, sex- and, sampling date-adjusted prevalence of anti-SARS-CoV-2 IgG was significantly higher (Poisson model RR [95\% Cl] 2.36 [1.03-5.43], $\mathrm{p}=0.043)$ in the $\mathrm{HC}$ control group $(4.21 \%$; prevalence [95\% Cl] 0.042 [0.022 to 0.074$]$ ), representing subjects from the clinics and practices treating IMID patients. Unexpectedly, only 4 out 534 IMID patients $(0.75 \%$; prevalence [95\% CI] 0.007 [0.002 to 0.019]) developed anti-SARS-CoV-2 IgG responses. In the age-, sex- and, sampling-date-adjusted Poisson model prevalence of anti-SARS-CoV-2 IgG responses was significantly lower (RR 0.32 [0.11 to 0.99], $p=0.048$ ) compared to reference NHC control group, despite that all of these patients received continuous treatment with cytokine blocking agents. In contrast, IMID patients receiving no cytokine blockade had similar (RR 1.21 [0.50 to 2.90], $p=0.676$ ) prevalence of anti-SARS-CoV-2 Ig responses compared to HC controls (3.1\%; prevalence [95\% Cl] 0.031 [0.013 to 0.061]). Details on the breakdown of seropositivity stratified by group, age, sex and other covariates are shown in Suppl. Table 1.

Positive IgG responses against the SARS-CoV-2 S1 domain were validated by two independent tests, one chemo-luminescence assay for Ig G against the spike and nucleocapsid protein and an enzyme-linked immunosorbent assay for IgG against the nucleocapsid protein only (Figure 1C). Furthermore, the pattern of immune responses against the spike protein S1 domain, the receptor binding domain of the S1 domain, the extracellular domain of the S2 domain and the nucleocapsid of SARS-CoV-2 were identical in the positively tested samples and patients with RNA proven COVID-19 but different from patients with endemic HCoV infection (Figure 1D). These data indicate that anti-SARS-CoV-2 IgG responses are derived from COVID-19 but not endemic HCoV infections.

Our data are consistent with the idea that IMID patients treated with cytokine inhibitors show reduced susceptibility to SARS-CoV-2 infection and COVID-19. This finding is at first sight counterintuitive as IMIDs are associated with an inherently enhanced infection risk, also pertaining to viral infections [9]. Furthermore, inhibition of some of the pro-inflammatory cytokines has shown to increase the risk for infections, in particular bacterial and fungal infections [10]. Nonetheless, no increased risk for viral infections, i.e., respiratory infections such as influenza has been reported with the inhibition of TNFa-, IL-6, IL-17 or IL-23, which are the most frequent cytokine inhibitors currently used for the treatment of IMIDs [11]..

The low seroprevalence of SARS-CoV-2 infections in anti-cytokine treated IMIDs could have two principle explanations: While (i) the four groups were recruited in the same region, (ii) the $\mathrm{HC}$ control group having the highest prevalence for SARS-CoV-2 IgG was in direct contact with the IMID patients and (iii) all subjects were exposed to similar detailed information regarding social behavior during the outbreak of the COVID-19 pandemic in Germany, IMID patients may have followed an even more stringent exposure prophylaxis than controls. We rigorously tested this hypothesis and showed that deviation from expected frequencies of social contacts and behavior of IMID patients with and without cytokine inhibitors was very similar (Figure 1E), while, not unexpectedly, HC 
controls showed a pattern of higher exposure risk and higher frequency of symptoms (Suppl. Table 2). Since, seroprevalence of SARS-CoV-2 infection of IMID patients without cytokine inhibitors was similar to controls, other factors need to be considered that account for the very low seroprevalence of SARS-CoV-2 infection in cytokine inhibitor-treated IMID patients.

Two recent studies reported that development of a more pronounced anti-SARS-CoV-2 IgG response is linked to more severe disease, suggesting inflammatory responses as important triggers for priming adaptive immunity against SARS-CoV-2 [12,13]. Similar observations have been made for SARSCoV [14]. Hence, tissue injury and the related release of pro-inflammatory mediators, which drive the symptoms of COVID-19, may also initiate robust antibody responses. Infection of epithelial cells by SARS viruses triggers the release of cytokines such as TNFa and IL-6 [15], which are key cytokines in IMIDs, where they are known to aggravate inflammation and tissue damage. Therefore, treatment with cytokine blockers may dampen the inflammatory tissue damage in response to SARS-CoV-2, limit clinical onset of COVID-19 and also inhibit the formation of anti-SARS-CoV-2 antibodies. Not surprisingly, cytokine blocking strategies, such as IL-6 inhibition, are currently tested for the treatment of the hyper-inflammatory syndrome associated with COVID-19 [16].

Although we have extensively validated anti-SARS-CoV-2 antibody responses it can't be fully excluded that some of the responses are due to cross-reacting antibodies raised by endemic coronavirus infections. If this was the case our data would indicate that IMID patients treated with cytokine inhibitors also have a reduced susceptibility to infections with endemic coronaviruses. Furthermore, virtual absence and/or mild clinical course of clinical COVID-19 infection in IMID patients [17-20] argues for reduced susceptibility of these patients to SARS-CoV-2 and supports the concept of a protective or at least mitigating effect of treatment with cytokine-blocking drugs.

In summary, these data suggest that patients with IMIDs receiving cytokine inhibitors are not at enhanced but at lower risk for SARS-CoV-2 infection compared to the general community and IMID patients not receiving such drugs. These data support a central pathogenic role of cytokines in COVID-19 and clearly speaks against stopping cytokine inhibitor treatment in patients with IMIDs during the current SARS-CoV-2 pandemic.

\section{Online Methods}

\section{Patients}

Patients with immune-mediated inflammatory diseases (IMID; $\mathrm{N}=534$ ) were recruited in centers in Erlangen, Nuremberg and Bamberg (FAU ErlangenNuremberg, IMID practices in Erlangen and Bamberg). These centers are specialized in the treatment of IMID patients in the field of rheumatology, gastroenterology and dermatology. Patients were offered to participate if they were on stable (> 3 months) treatment with cytokine inhibitors, comprising either therapeutic antibodies and receptors (such as those neutralizing tumor necrosis factor alpha or interleukins- $6,-17$ or -23 ) or chemical agents (such as Janus Kinase inhibitors). In addition, a cohort of IMID patients $(\mathrm{N}=259)$ receiving no cytokine inhibitors within the last three months was recruited in the same centers. Center distribution of IMID patients with and without cytokine inhibitors was identical.

\section{Controls}

In addition, two control groups were analyzed. The first ( $\mathrm{N}=285)$ included health care $(\mathrm{HC})$ professionals (doctors, nurses and technicians), who work in the aforementioned institutions and are involved in the treatment, diagnostics and research on IMID patients. Subjects were contacted personally as well as by email and invited to participate in the study. None of $\mathrm{HC}$ professionals denied participating in this study as their interest in receiving coronavirus diagnostics was very high. In addition and since the exposure of the $\mathrm{HC}$ control to infectious agents may be higher than in the general population, an additional control group with healthy subjects not involved in health care was analyzed ("non-health care control", NHC; N=971). This group was composed of two populations: A cohort of healthy subjects $(\mathrm{N}=329)$ from the district of Erlangen-Höchstadt and the city of Erlangen, that has been established via field campaigns to assesses healthy ageing. These subjects were not allowed to have a diagnosis of any IMID. Part of this cohort has been described previously (Berlin A, et al. Osteoarthritis Cartilage. 2019;27:1043-1047) and numbers have been expanded since then. The second part was a cohort of firefighters ( $\mathrm{N}=651$ ) from the same region (district of Erlangen-Höchstadt and the city of Erlangen), which was recruited by an organized field campaign in March and April 2020.

Recruitment was centrally organized by the volunteer fire brigade officers, who invited all firefighters of their sub-units to participate (via the corresponding fire brigade mailing lists). All firefighters agreed to participate in this study. As the prevalence of anti-SARS-Cov2 antibodies was very similar in the non-firefighter cohort (2.12\%) and the firefighter cohort (2.36\%.), the two NHC control cohorts were pooled.

\section{Ethical approval}

Ethical approval (\#157_20 B) to conduct this analysis was granted by the institutional review board (IRB) of the University Clinic of Erlangen. Written informed consent was obtained from the study participants.

\section{Demographic characteristics}

Age, sex and body mass index were documented in all patients and controls. In addition, history of smoking, arterial hypertension, diabetes mellitus and chronic lung diseases, which put patients at risk for severe COVID-19 infection were documented.

\section{Assessment of symptoms and social contacts}


The study groups were exposed to questions on clinical symptoms (cough, rhinitis, throat pain, fever, headache, fatigue, musculoskeletal pain, anosmia, shortness of breath and diarrhea) and social contacts (contact with infected individuals, travel to at risk areas, working outside home) provided by the German federal authority for infectious diseases (Robert Koch Institute) and disseminated through routes of public communication, as well as social distancing rules imposed by the government at the beginning of the study period. Data about symptoms and social contacts were self-documented (present/absent, complied/not-complied) using a standardized form during the study period (February $1^{\text {st }}$ to April $15^{\text {th }}, 2020$ ). These data were self-documented and retrieved when serum analysis was done after 8 weeks.

\section{Anti-SARS-CoV-2 antibody testing}

Serum samples were taken between March $18^{\text {th }}$ and April $30^{\text {th }}$ for anti-SARS-CoV-2 IgG tests. Immunoglobulin G (IgG) antibodies against the S1 domain of the spike protein of SARS-CoV-2 where tested by the recent CE version (April 2020) of the commercial enzyme-linked immunosorbent assay from Euroimmun (Lübeck, Germany) using the EUROIMMUN Analyzer I platform and according to the manufacturers protocol. Optical density was determined at $450 \mathrm{~nm}$ with reference wavelength at $630 \mathrm{~nm}$. A cut-off of $\geq 0.8$ (OD450nm) was considered as positive. This assay has very high (>99\%) specificity (see also https://www.ukbonn.de/

C12582D3002FD21D/vwLookupDownloads/Streeck_et_al_Infection_fatality_rate_of_SARS_CoV_2_infection2.pdf/\%24FILE/Streeck_et_al_Infection_fatality_rat and has been approved for diagnostic use in the United States (see https://www.centerforhealthsecurity.org/resources/COVID-19/serology/Serology-basedtests-for-COVID-19.html\#sec2. Assays were performed in line with the guidelines of the German Medical Association (RiliBAK) with stipulated internal and external quality controls.

\section{Tests used for validation of positive results}

Two reference tests for IgG antibodies against the SARS-CoV-2 were used, one of them a chemo-luminescent assay (CLIA) for detecting antibodies against the spike and nucleocapsid proteins (Shenzhen Yhlo Biotech, iFlash-SARS-CoV-2, Cat \#C86095G, Shenzhen, China). This assay is based on magnetic beads coated with SARS-CoV-2 spike and nucleocapsid antigen for the detection of specific IgG using a fully automated iFlash Immunoassay Analyzer (Shenzhen Yhlo Biotech). The assays were performed according to the manufacturer's protocols. The lgG titre was automatically calculated as arbitrary units (AU/ml) and the cut-off value for a positive test was $10 \mathrm{AU} / \mathrm{ml}$. In addition, an enzyme-linked immunosorbent assay detecting antibodies against the nucleocapsid protein (Immundiagnostik, Bensheim, Germany). Optical density was determined at $450 \mathrm{~nm}$ with reference wavelength at $630 \mathrm{~nm}$. A cut-off of $\geq 0.5$ (OD $450 \mathrm{~nm}$ ) was considered as positive.

\section{In-house SARS-CoV-2 ELISA}

An in-house ELISA was prepared according to published coating protocols (ref. 1). In detail, $100 \mu$ l of the coating solution, consisting of $1.5 \mu \mathrm{g} / \mathrm{ml}$ of one of the above indicated SARS-CoV-2 antigens in 1 XPBS, were applied to wells of a ninety-six well plates (Costar), incubated overnight at $4^{\circ} \mathrm{C}$. The following antigens were used: recombinant SARS-CoV-2 Spike Protein, S1 Subunit (1-Us-Tag); SARS-CoV-2 Spike S1 receptor binding domain (RBD) (His-Tag) (both Sino Biological, Beijing, China); SARS-CoV-2 (201 9-nCoV) Meridian Biosciences (Memphis, TX); Spike Protein (S2 ECD, His tag); SARS-CoV-2 (COVID-19) nucleocapsid protein(Sino Biological, Beijing, China). After removing the coating solution Liquid plate sealer animal free (\#163050, Candor, Germany) was added to the plates at room temperature (RT) for 1 hour as blocking solution. Serum samples diluted 1:100 in 1\% bovine serum albumin in $1 \times$ TBS/0,01\% Tween were added to the plates for $30 \mathrm{~min}$ at room temperature. The following samples were used: (1) Controls with anti-SARS-CoV-2 IgG negative IgG in the Euroimmun ELISA ( $\mathrm{N}=6)$, (2) Controls with anti-SARS-CoV-2 IgG positive IgG in the Euroimmun ELISA (N=6), (3) patients with COVID-19 infection and positive viral RNA test $(\mathrm{N}=6)$, patients with endemic human coronavirus infection during the pre-SARS-CoV-2 era in spring 2019 ( $N=5)$. Plates were washed three times with 200 ul per well of 1xTBS/0.01\% Tween. Next, a 1:15.000 dilution of goat anti-human IgG-horseradish peroxidase (HRP) conjugated secondary antibody (Dianova) was prepared in HRP-Protector solution (\#222 050, Candor, Germany) and added to the wells for 15 min. Plates were again washed thrice with 1xTBS/0.01\% Tween. Once completely dry, 100 ul of 3, 3',5,5'-Tetramethylbenzidine Liquid (TMB, Sigma Aldrich) Substrate solution was added to each well of the plates for 15 minutes and then the reaction was stopped by addition of $100 \mu \mathrm{L}$ per well of $0.16 \mathrm{M}$ sulfuric acid. Absorbance was measured at $450 \mathrm{~nm}$. The positive cut off was equal to the mean of the $\mathrm{OD}$ values of the negative control wells on the respective plate plus three times the standard deviation of the $\mathrm{OD}$ value distribution from the negative control samples.

\section{Statistics}

We summarized participant characteristics using means, standard deviations and percentages as appropriate. For anti-SARS-CoV2 IgG positivity ( $\geq 0.8$ OD450nm) exact $95 \%$ confidence intervals were constructed based on the Poisson approximation to the binomial distribution. We estimated relative risks of seropositivity in study groups using the NHC group as the reference and adjusting for age, sex and sampling-date using a Poisson regression model with robust sandwich standard errors (ref. 2). Adjustment for sampling date was achieved using the cumulative confirmed COVID-19 case-counts reported by the Robert Koch Institute for Erlangen and Erlangen-Höchstadt on the date of serum sampling

(https://experience.arcgis.com/experience/478220a4c454480e823b17327b2bf1d4; accessed on 07.05.2020 at 16.24). We reasoned that these case counts would approximate the overall risk of exposure to SARS-CoV-2 from the onset of the pandemic to the date of sampling. We constructed $4 \times 2$ contingency tables for binary categories of (i) patient-reported history of contact with persons having a febrile respiratory tract infection, (ii) absence from workplace, such as working from home, being unemployed or retired, and (iii) travel to high-risk regions. Using these contingency tables we calculated and plotted standardized Pearson's residuals showing the deviation of the observed frequencies in each study group from expected frequencies of the relevant category. Results for 
chemo-luminescent detection of anti-S1 spike protein antibodies (Yhlo Biotech) and anti-nucleocapsid antibodies (Immunodiagnostik) were compared between S1-positive and S1-negative subjects using Mann Whitney U test. A p value of less than 0.05 was considered as significant. All analyses were done using R v.3.5.3 software (R Foundation for Statistical Computing, Vienna, Austria) and GraphPad Prism v8.1 (GraphPad Software, San Diego, USA).

\section{Data availability}

Anonymized raw data and $\mathrm{R}$ syntax to reproduce the analyses, figures, tables in the published article are available.

\section{Methods References}

1. Amanat F, Stadlbauer D, Strohmeier S, et al. A serological assay to detect SARS-CoV-2 seroconversion in humans. medRxiv 2020:2020.03.17.20037713.

2. Barros, A.J. \& Hirakata, V.N. Alternatives for logistic regression in cross-sectional studies: an empirical comparison of models that directly estimate the prevalence ratio. BMC Medical Research Methodology 2003;3, 21. doi: 10.1186/1471-2288-3-21.

\section{Declarations}

Competing interest: There is NO competing interest.

\section{References}

[1] Wu F., et al. Nature 2020; 579: 265-269.

[2] Huang C, et al. Lancet 2020;395: 497-506.

[3] Schett G, et al. Nat Med 2013, 19: 822-4.

[4] Pedersen SF, Ho YC. J Clin Invest. 2020 Mar 27. pii: 137647

[5] Berlin A, et al. Osteoarthritis Cartilage. 2019;27:1043-1047.

[6] Meyer B, Drosten C, Muller MA. Virus Res. 2014;194:175-83.

[7] Petherick A. Lancet 2020;395:1101-02.

[8] Okba NMA, et al. medRxiv; doi.org/10.1101/2020.03.18. 20038059

[9] Tinsley A, et al. Inflamm Bowel Dis 201925:369-376.

[10] Winthrop KL, et al. Ann Rheum Dis. 2015 Dec;74:2107-16.

[11] Blumentals WA et al., BMC Musculosskelet Disord 2012;13:158

[12] Zhang B et al. medRxiv 2020; doi.org/10.1101/2020.03.12.20035048

[13] Zhao, J et al. Clin. Infect. Dis 2020; https://doi.org/10.1093/cid/ciaa344

[14] Peiris JS, et al. Lancet, 2003. 361:1767-72.

[15] Yoshikawa T, et al. J Virol. 2009; 83: 3039-3048.

[16]Luo P, et al. J Med Virol. 2020 Apr 6.

[17]An P, et al. Lancet 2020; https://ssrn.com/abstract=3543590

[18] Norsa L, et al. Gastroenterology 2020 doi.org/10.1053/j.gastro.2020.03.062

[19] Monti S, et al. Ann Rheum Dis 2020; doi: 10.1136/annrheumdis-2020-217424.

[20] Haberman R, et al. N Engl J Med 2020; DOI: 10.1056/NEJMc2009567.

\section{Figures}




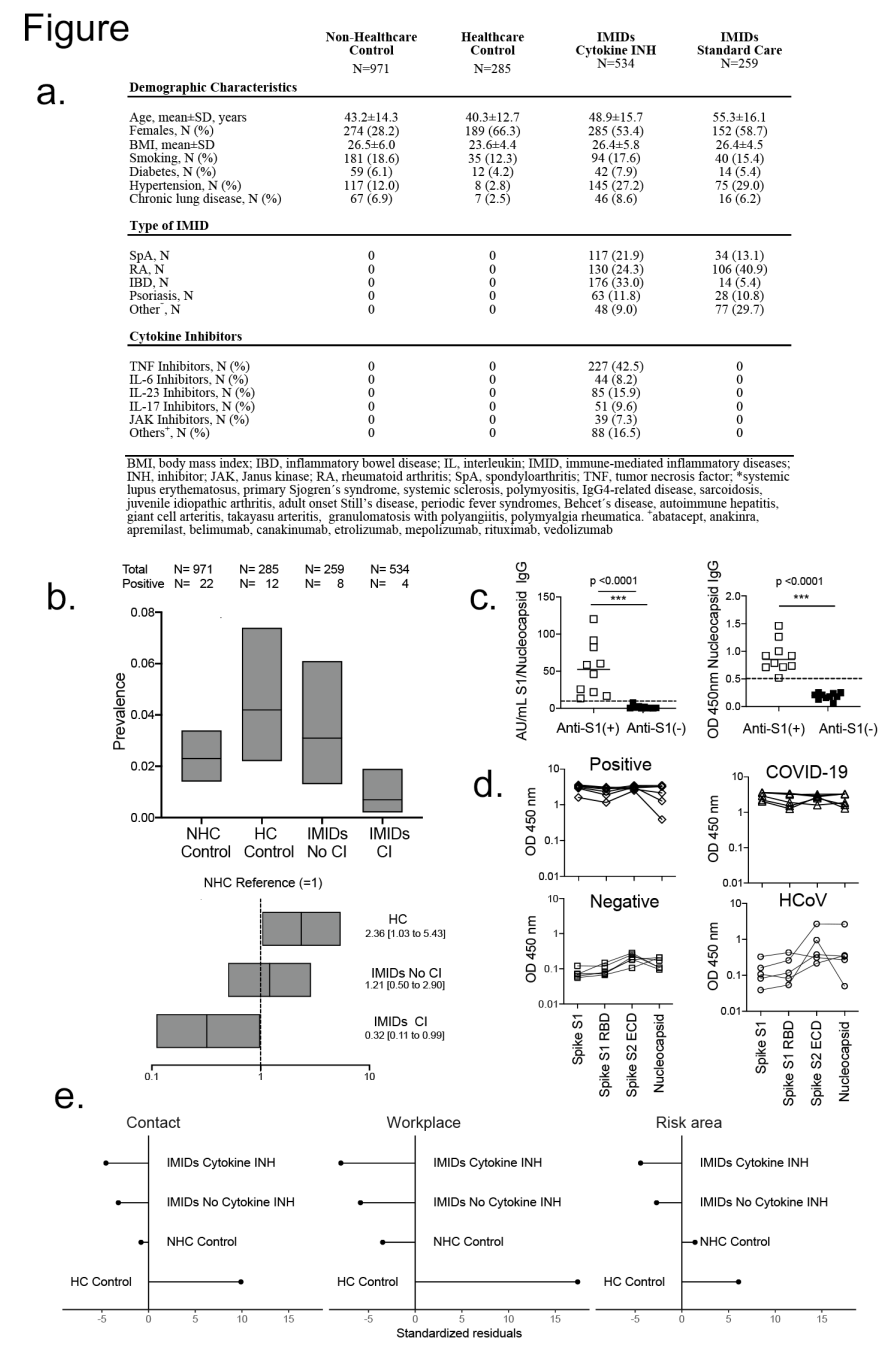

\section{Figure 1}

(a) Characteristics of the patients with IMIDs and controls. (b) Top: Prevalence and 95\% confidence intervals of a positive anti-SARS-CoV-2 IgG antibody test recognizing the $\mathrm{S} 1$ domain of the spike protein in non-health care $(\mathrm{NHC})$ controls, health care $(\mathrm{HC})$ controls and IMIDs with and without cytokine inhibitors (CI); bottom: risk ratios and 95\% confidence intervals of anti-SARS-CoV-2 IgG antibody positivity in health care (HC) controls and IMIDs with and without cytokine inhibitors (Cl) with $\mathrm{HC}$ controls as reference; (c) Comparison of anti- SARS-CoV-2 IgG positive (anti-S1+; N=10) and negative (anti-S1-; N=10) samples (in Euroimmune ELISA) for reactivity in the chemi-luminescent anti-SARS-CoV-2 Spike S1/nucleocapsid IgG test (Yhlo Biotech) and anti-nucleocapsid IgG antibody ELISA (Immundiagnostik Inc). (d) Validation with in-house ELISA testing reactivities against (1) the S1 domain of the spike protein, (2) the receptor binding domain (RBD) of the S1 domain of the spike protein, (3) extracellular domain (ECD) of the S2 domain of the spike protein and (4) the nucleocapsid in anti-SARS-CoV-2 IgG negative ( $\mathrm{N}=6)$ and positive $(\mathrm{N}=6)$ samples (in Euroimmune ELISA), COVID-19 patients with positive viral RNA test ( $\mathrm{N}=6)$ and subjects with endemic human coronavirus ( $\mathrm{HCoV}$ ) infection $(\mathrm{N}=5)$ in the pre- SARS-CoV-2 time. (e) Standardized Pearson's residuals showing deviation from the expected frequencies for exposure risk variables (contact with persons with a respiratory infection, presence at workplace outside home, travel to risk areas) of IMID patient groups and control groups.

\section{Supplementary Files}

This is a list of supplementary files associated with this preprint. Click to download.

- Supplementarytable1.pdf

- Supplementarytable2.pdf 